\title{
Selenium-integrated multi-resonance induced TADF emitters boost EQE of electroluminescence to $40.5 \%$ with ultra-low efficiency roll-off
}

\author{
Yu Xuan Hu \\ Shenzhen University \\ Jingsheng Miao \\ Shenzhen University \\ Tao Hua \\ Shenzhen University

\section{Zhongyan Huang} \\ Shenzhen University \\ Yanyu Qi \\ Shenzhen University \\ Yang Zou \\ Shenzhen University \\ Yuntao Qiu \\ Shenzhen University \\ Han Xia \\ Shenzhen University \\ He Liu \\ Shenzhen University \\ Xiaosong Cao \\ Shenzhen University \\ Chuluo Yang ( $\nabla$ clyang@szu.edu.cn ) \\ Shenzhen University
}

\section{Article}

Keywords:

Posted Date: February 9th, 2022

DOI: https://doi.org/10.21203/rs.3.rs-1145606/v1

License: () (i) This work is licensed under a Creative Commons Attribution 4.0 International License. Read Full License

Version of Record: A version of this preprint was published at Nature Photonics on October 13th, 2022. See the published version at https://doi.org/10.1038/s41566-022-01083-y. 


\section{Abstract}

Multi-resonance induced thermally activated delayed fluorescent (MR-TADF) materials have shown great potential in high-efficiency and narrowband organic light-emitting diodes (OLEDs). However, obvious efficiency roll-off attributed to slow reverse intersystem crossing (RISC) process hinders MR-TADF materials from practical applications. Here, we report a heavy-atom incorporating emitter, namely BNSeSe, based on selenium-integrated boron-nitrogen skeleton, showing $100 \%$ photoluminescence quantum yield and the highest rate of RISC ( $k_{\text {RISC }}$ ) of $2.0 \times 10^{6} \mathrm{~s}^{-1}$ among MR-TADF molecules. The corresponding OLEDs exhibit excellent external quantum efficiency (EQE) up to $36.8 \%$ and ultra-low roll-off character at high brightness (with very small roll-off values of $2.8 \%$ and $14.9 \%$ at $1000 \mathrm{~cd} \mathrm{~m}^{-2}$ and $10000 \mathrm{~cd} \mathrm{~m}^{-2}$, respectively). Furthermore, the outstanding capability to harvest triplet excitons also enables BNSeSe to be a superior sensitizer for hyperfluorescence (HF) device, which shows state-of-the-art performance with record high EQE of 40.5\%, power efficiency (PE) beyond $200 \mathrm{Im} \mathrm{W}^{-1}$ and luminance close to $200000 \mathrm{~cd} \mathrm{~m}^{-2}$.

\section{Full Text}

Organic light-emitting diodes (OLEDs) with simultaneously high efficiency and narrowband emission become increasingly important for the demands on energy-saving and high-quality of displays. Thanks to the pioneer work by Hatakeyama et al., multi-resonance (MR) thermally activated delayed fluorescent (TADF) emitters have emerged with the astonishing narrowband emission that could fulfill the requirements. ${ }^{1,2} \mathrm{~A}$ high external quantum efficiency (EQE) up to $34 \%$ and electroluminescence (EL) with full width at half maximum (FWHM) of $18 \mathrm{~nm}$ demonstrated their great potential towards practical applications. ${ }^{3}$ However, MR-TADF emitters usually possess long delay lifetime of several tens of microsecond, which usually leads to large efficiency roll-off at high brightness and thus impede their commercialization. ${ }^{4-10}$

To reduce efficiency roll-off, both singlet and triplet excitons should be converted expeditiously. The intrinsic large Frank-Condon overlap integral ensures MR-TADF emitters with fast radiative rate of singlet excitons. While the recycle of triplet ones depends on the reverse intersystem crossing (RISC) channel, so the rate of reversed intersystem crossing $\left(k_{\mathrm{RISC}}\right)$ becomes the key factor to realize low efficiency roll-off. ${ }^{11-14}$ According to Fermi's Golden rule, $k_{\mathrm{RISC}}$ between two states is described as ${ }^{15-18}$

$$
k_{\mathrm{RISC}} \propto\left|\left\langle\mathrm{S}\left|\hat{\mathrm{H}}_{\mathrm{SOC}}\right| \mathrm{T}\right\rangle\right|^{2} \exp \left(\frac{-\Delta E_{\mathrm{ST}}}{k_{\mathrm{B}} \mathrm{T}}\right)
$$

where $\left\langle\mathrm{S}\left|\hat{H}_{\mathrm{SOC}}\right| \mathrm{T}>\right.$ is the spin orbital coupling (SOC) matrix element, and $\Delta E_{\mathrm{ST}}$ is the energy difference between the corresponding states. To enhance $k_{\mathrm{RISC}}$, one of the practicable strategies is narrowing the $\Delta E_{\mathrm{ST}}$ to provide a smaller energy gap benefiting upconversion process from low-energy $T_{1}$ to high-energy $S_{1}$ state. ${ }^{19,20}$ Generally, $\Delta E_{S T}$ can be reduced via minimizing the overlap of the highest occupied molecular orbital (HOMO) and the lowest unoccupied molecular orbital (LUMO) which are already atomically separated in MR-TADF emitters. Such characteristic leaves little room to reduce the value. Thereby, enhancing SOC could be a more practical strategy to facilitate RISC. Heavy-atom effect (HAE) has been verified to be an effective method to enhance SOC in phosphorescent emitters. ${ }^{21-24}$ In our previous work, sulfur has been introduced into MR-TADF system which shortens the lifetime of delayed fluorescence $\left(\tau_{d}\right)$ and smooths the efficiency roll-off compared to its oxygen substituted analog, but the device performance is still far less than satisfactory. ${ }^{25}$ Besides optimizing from the molecular level, an alternative TADF sensitizing strategy, also known as hyperfluorescence, has been demonstrated to be effective to achieve OLEDs with excellent performance, where a TADF emitter is used as sensitizer to recycle the triplet excitons and then transfer the singlet excitons to the terminal emitter. ${ }^{26-28}$ The long-range Förster resonance energy transfer (FRET) interaction within this system endows dopant-concentration-dependent energy transfer rates with high values of $10^{8}-10^{11} \mathrm{~s}^{-1}$, which benefits a quick consumption of triplet excitons to suppress exciton annihilation, such as triplet-triplet annihilation (TTA), triplet-polaron annihilation (TPA) and singlet-triplet annihilation (STA). ${ }^{29-34}$. The core of this mechanism lies on the fast harvesting of triplet excitons via the TADF sensitizer, which also demands a fast RISC process. ${ }^{28,35,36}$ In this context, we are driven to develop MR-TADF emitters with fast RISC which not only facilitates their emissive properties as emitters but also ensures them as excellent sensitizers for highly efficient hyperfluorescence (HF) OLEDs. 


\section{Results}

Molecular design and theoretical calculations. The molecules design in this contribution are presented in Fig. 1a. Conventional boron-nitrogen skeleton was chosen for evaluation due to their high photoluminescence quantum yield $\left(\Phi_{\mathrm{PL}}\right)$ and narrowband emission. Selenium ( $S e, Z_{N}=34$ ) is inserted to enhance the SOCs in BNSSe and BNSeSe. The asymmetric BNSSe is developed to establish a fine comparison and comprehend the structure-property relationship. Their oxygen and sulfur counterparts (2PXZBN, 2PTZBN) are also prepared to fully validate our molecular design.

We first used density functional theory (DFT) under the representative B3LYP/6-31G(d,p) set to optimize their ground state geometries. All these four molecules show well separated HOMO and LUMO distribution. The more twisted structures see Fig. S11 of BNSSe and BNSeSe (compared to 2PXZBN and 2PTZBN) may induce them with less denser packing to avoid inter-chromophore interactions. According to the time-dependent DFT (TD-DFT) analysis, high oscillator strength values beyond 0.2 are acquired in these four emitters, implying a fast radiative decay. Small $\Delta E_{\mathrm{ST}} \mathrm{S}$ are also observed, suggesting their potential TADF activity. To evaluate the internal heavy atom effect, the SOCMEs of $S_{1}-T_{1}, S_{1}-T_{2}$ and $S_{1}-T_{3}$ for them were further predicted by PySOC method. As depicted in Fig. 1a, both 2PXZBN and 2PTZBN show small SOC values. 2PXZBN and 2PTZBN exhibit very tiny SOCs between $\mathrm{S}_{1}$ and $\mathrm{T}_{1}\left(\left\langle\mathrm{~S}_{1}\left|\hat{H}_{\text {Soc }}\right| \mathrm{T}_{1}\right\rangle=0.070\right.$ and $0.030 \mathrm{~cm}^{-1}$, respectively). In sharp contrast, significant enhancements are achieved in seleniumcontained BNSSe and BNSeSe $\left(<S_{1}\left|\hat{H}_{\text {Soc }}\right| T_{1}>=4.654\right.$ and $6.043 \mathrm{~cm}^{-1}$, respectively). SOCs between $S_{1}$ and $T_{n}(n=2$ or 3$)$ for BNSSe and BNSeSe are also much larger than those for 2PXZBN and 2PTZBN. $\left\langle\mathrm{S}_{1}\left|\hat{H}_{\mathrm{SOC}}\right| \mathrm{T}_{2}>\right.$ of $84.46 \mathrm{~cm}^{-1}$ and $\left\langle\mathrm{S}_{1}\left|\hat{H}_{\mathrm{SoC}}\right| \mathrm{T}_{3}>\right.$ of $95.75 \mathrm{~cm}^{-1}$ for BNSSe are obtained, which are dozens of times higher than its sulfur analogue (2PTZBN, $\left\langle S_{1}\left|\hat{H}_{\text {SOC }}\right| T_{2}\right\rangle=1.003 \mathrm{~cm}^{-1}$ and $\left\langle S_{1}\right|$ $\hat{H}_{\text {SoC }} \mid T_{3}>=1.351 \mathrm{~cm}^{-1}$ ). Even better, $\left\langle\mathrm{S}_{1}\left|\hat{H}_{\text {SoC }}\right| \mathrm{T}_{2}>\right.$ and $\left\langle\mathrm{S}_{1}\left|\hat{H}_{\text {SoC }}\right| \mathrm{T}_{3}>\right.$ for BNSeSe dramatically increase to 112.7 and $235.1 \mathrm{~cm}^{-1}$, respectively, which are almost comparable to the SOC constants for some phosphorescent emitters involving noble metals. ${ }^{37,38}$ These results theoretically support our molecular design, suggesting that the introduction of selenium may immensely promote the SOCs and subsequently accelerate the RISC processes.

Table 1 | Physical data and kinetic parameters 2PXZBN, 2PTZBN, BNSSe and BNSeSe in 1wt\% DMIC-TRZ doped film.

\begin{tabular}{|llllllllllll|}
\hline & $\begin{array}{c}\lambda_{\mathrm{PL}} \\
{[\mathrm{nm}]}\end{array}$ & $\begin{array}{c}\mathrm{S}_{1} \\
{[\mathrm{eV}]}\end{array}$ & $\begin{array}{c}\mathrm{T}_{1} \\
{[\mathrm{eV}]}\end{array}$ & $\begin{array}{c}\Delta E_{\mathrm{ST}} \\
{[\mathrm{eV}]}\end{array}$ & $\begin{array}{c}\Phi_{\mathrm{PL}} \\
{[\%]}\end{array}$ & $\begin{array}{c}\Phi_{\mathrm{TADF}} \\
{[\%]}\end{array}$ & $\begin{array}{c}\tau_{\mathrm{PF}} \\
{[\mathrm{ns}]}\end{array}$ & $\begin{array}{c}\tau_{\mathrm{DF}} \\
{[\mu \mathrm{s}]}\end{array}$ & $\begin{array}{c}k_{\mathrm{r}, \mathrm{S}} \\
{\left[10^{7} \mathrm{~s}^{-1}\right]}\end{array}$ & $k_{\mathrm{ISC}}$ & $k_{\mathrm{RISC}}$ \\
\hline 2PXZBN & 523 & 2.54 & 2.39 & 0.15 & 71 & 28 & 5.2 & 38.1 & 8.2 & 0.75 & 0.043 \\
\hline 2PTZBN & 525 & 2.55 & 2.42 & 0.13 & 91 & 68 & 5.1 & 20.7 & 4.5 & 1.5 & 0.19 \\
\hline BNSSe & 520 & 2.56 & 2.44 & 0.12 & 99 & 86 & 3.0 & 12.7 & 4.3 & 2.9 & 0.60 \\
\hline BNSeSe & 514 & 2.58 & 2.44 & 0.14 & 100 & 95 & 1.9 & 9.9 & 2.6 & 4.9 & 2.0 \\
\hline
\end{tabular}

Crystallographic and photophysical properties. As shown in crystal structures of BNSSe and BNSeSe, molecules exhibit rigid configurations, which are beneficial to suppress non-radiative transition. (Fig. S12 and S13) Due to the folded configurations of phenothiazine and phenoselenazine units, molecules in crystals of BNSSe and BNSeSe are twisted and loosely stacking. The photophysical properties of BNSSe and BNSeSe including absorption, fluorescence at $298 \mathrm{~K}$ and phosphorescence spectra at $77 \mathrm{~K}$ were measured in toluene as collected in Fig. 2a and Table S5. BNSSe and BNSeSe show intense absorption bands at $469 \mathrm{~nm}$ and $467 \mathrm{~nm}$, respectively, which refer to intramolecular charge transfer (ICT) processes. Both compounds exhibit green emission with fluorescence spectra peaked at $505 \mathrm{~nm}$ and $502 \mathrm{~nm}$ for BNSSe and BNSeSe, respectively. To evaluate the $\Delta E_{\mathrm{ST}}$ values of these materials, the lowest singlet and triplet excited-state energies were estimated from the onsets of fluorescence and phosphorescence bands at $77 \mathrm{~K}$. BNSSe and BNSeSe display equal $\Delta E_{S T}$ values at $0.17 \mathrm{eV}$, and these small energy gaps are beneficial for RISC from triplet excited states to singlet excited state. Prompt fluorescence $\left(\tau_{P F}\right)$ and delayed fluorescence $\left(\tau_{D F}\right)$ lifetimes of BNSSe are $1.6 \mathrm{~ns}$ and 2.1 $\mu$ s in oxygen-free toluene, respectively, which are distinctly smaller than those of 2PXZBN and 2PTZBN. Moreover, BNSeSe 
possesses a further decreased $\tau_{P F}$ of $0.76 \mathrm{~ns}$ and $\tau_{D F}$ of $1.0 \mu \mathrm{s}$, which are shortest among all reported MR-TADF emitters. Such short delayed fluorescence lifetimes are beneficial for utilizing excitons and suppressing efficiency roll-off. To gain a deeper insight into the emission in solid state, the photophysical properties for $1 \mathrm{wt} \%$-doped films of 2PXZBN, 2PTZBN, BNSSe and BNSeSe were recorded in 1,3-dihydro-1,1-dimethyl-3-(3-(4,6-diphenyl-1,3,5-triazin-2-yl)phenyl)indeno-[2,1-b]carbazole (DMIC-TRZ), a universal host featuring bipolar charge transport ability. As depicted in Fig. 2c, all four doped films exhibit green emission with slight bathochromic-shifts with respect to their toluene solution. PL decay curves of those films were shown in Fig. $2 d$. Both $\tau_{P F}$ and $\tau_{D F}$ drop in the sequence of $2 \mathrm{PXZBN}>2 \mathrm{PTZBN}>\mathrm{BNSSe}>\mathrm{BNSeSe}$. Furthermore, $\Phi_{\mathrm{PL}} \mathrm{S}$ of 2PXZBN, 2PTZBN, BNSSe and BNSeSe were measured to be $71 \%, 91 \%$, $99 \%$ and $100 \%$, respectively, with obvious escalating trend as $\tau_{\mathrm{DF}}$ shortened. The radiative decay rate constants of fluorescence $\left(k_{\mathrm{r}, \mathrm{S}}\right)$, intersystem crossing $\left(k_{\text {ISC }}\right)$ and $k_{\mathrm{RISC}}$ are further analyzed using a method provided in the literature based on these lifetimes and quantum yields. With the increasing number of Se atoms, the $k_{\text {RISC }}$ is greatly enhanced from $6.0 \times 10^{5} \mathrm{~s}^{-1}$ of BNSSe to $2.0 \times 10^{6} \mathrm{~s}^{-1}$ of BNSeSe, noting the latter is the largest value among all MR-TADF emitters (typically in the order of $10^{3} \sim 10^{5} \mathrm{~s}^{-1}$ ) (see Table S7). Such tremendous enhancement on $k_{\mathrm{RISC}}$ is in line with the enhanced SOCs.

\section{OLED devices}

Given the prominent photoluminescent properties of 2PXZBN, 2PTZBN, BNSSe and BNSeSe, we fabricated and evaluated OLED devices A-D employing them as emitters, respectively, with the following device configuration: indium tin oxide (ITO)/1,1-bis[(di-4tolylamino)phenyl]cyclohexane (TAPC, $30 \mathrm{~nm}$ )/tris(4-carbazolyl-9-ylphenyl)amine (TCTA, $15 \mathrm{~nm}$ )/3,3-di(9H-carbazol-9-yl)biphenyl (mCBP, $10 \mathrm{~nm}$ )/EML (1 wt\% emitter in DMIC-TRZ, $50 \mathrm{~nm}$ )/(1,3,5-triazine-2,4,6-triyl)tris(benzene-3,1-diyl)tris(diphenylphosphine oxide) (POT2T, $20 \mathrm{~nm}$ )/1-(4-(10-([1,1'-biphenyl]-4-yl)anthracen-9-yl)phenyl)-2-ethyl-1 H-benzo[d]imidazole (ANT-BIZ, $30 \mathrm{~nm}) / 8$ hydroxyquinolinato lithium (Liq, $2 \mathrm{~nm}$ )/alumina (Al, $100 \mathrm{~nm}$ ). All the device data are collected and summarized in Table 2 and Table S6, and selectively presented in Fig. 3. Devices A-D (in which emitters are 2PXZBN, 2PTZBN, BNSSe and BNSeSe, respectively) all exhibit green electroluminescence peaked at 517, 520, 515 and $512 \mathrm{~nm}$, respectively. Narrowband emission with FWMHs of $50 \mathrm{~nm}$ and $48 \mathrm{~nm}$ were recorded for devices $\mathrm{C}$ and $\mathrm{D}$. Attributed to the host with dipole charge transport ability, all devices exhibit low turnon voltage below $2.5 \mathrm{~V}$ and extremely high luminance over $100000 \mathrm{~cd} \mathrm{~m}^{-2}$. All devices exhibit excellent performance such as high maximum EQEs $\left(\mathrm{EQE}_{\max }\right)$, power efficiencies $\left(\mathrm{PE}_{\mathrm{max}}\right)$ and current efficiencies $\left(\mathrm{CE}_{\max }\right)$. As depicted in Fig. 3c-3f, $E Q E_{\max } \mathrm{S}$ of $30.7 \%$, $34.6 \%, 35.7 \%$ and $36.8 \%$ were observed for devices $A-D$, respectively. $P E_{m a x} S$ of devices A-D are 123.4, 157.7, 156.2 as well as 146.3 Im $\mathrm{W}^{-1}$ and $C E_{\max } \mathrm{S}$ of them are high as $108.8,124.9,124.2$ and $121.0 \mathrm{~cd} \mathrm{~A}^{-1}$, respectively. To the best of our knowledge, PEs and CEs of devices based on 2PTZBN, BNSSe and BNSeSe are higher than almost all reported TADF OLEDs. Moreover, devices $\mathrm{C}$ and especially $D$ exhibit distinctly reduced efficiency roll-off compared to devices $A$ and $B$, which suggests that stronger SoCs between $S_{1}$ and $T_{n}(n=1,2,3)$ of BNSSe and BNSeSe suppress exciton annihilation more efficiently under high current density. It is worth mentioned that the efficiency roll-off of device $D$ is significantly smaller than that observed in the latest narrowband TADF OLEDs, 7,9 ,

${ }^{39-42}$ EQEs of device $D$ remain $34.0 \%$ at $1000 \mathrm{~cd} \mathrm{~m}^{-2}, 26.9 \%$ at $5000 \mathrm{~cd} \mathrm{~m}^{-2}$ and $21.9 \%$ at even $10000 \mathrm{~cd} \mathrm{~m}^{-2}$. We assume that the very strong SOC and subsequent high $k_{\text {RISC }}$ value suppress the triplet-involved annihilation processes in the device. (see Table S7) The operational lifetimes of devices A-D were preliminarily measured at an initial luminance of $1000 \mathrm{~cd} \mathrm{~m}^{-2}$. Device $A$ shows $L T_{50}$ of 158 h, nevertheless, while devices $B, C$ and $D$ exhibit shorter $L T_{50}$ of $5.6,4.8$ and 4.1 h, respectively.

\section{Table 2 | Summary of device performances for A-F.}




\begin{tabular}{|c|c|c|c|c|c|c|c|c|c|c|}
\hline \multirow{2}{*}{$\begin{array}{l}\text { Devices } \\
\text { (emitter) }\end{array}$} & \multicolumn{3}{|l|}{$\mathrm{CE}^{\mathrm{a}}\left[\mathrm{cd} \mathrm{A} \mathrm{A}^{-1}\right]$} & \multicolumn{3}{|l|}{$\mathrm{PE}^{\mathrm{b}}\left[\mathrm{Im} \mathrm{W} \mathrm{W}^{-1}\right]$} & \multicolumn{4}{|l|}{$\mathrm{EQE}^{\mathrm{C}}[\%]$} \\
\hline & $\begin{array}{l}\text { Max } \\
\text { (Average) }\end{array}$ & $\begin{array}{l}1000 \\
\mathrm{~cd} \mathrm{~m}_{2}^{-}\end{array}$ & $\begin{array}{l}10000 \\
\mathrm{~cd} \mathrm{~m}-2\end{array}$ & $\begin{array}{l}\text { Max } \\
\text { (Average) }\end{array}$ & $\begin{array}{l}1000 \\
\mathrm{~cd} \mathrm{~m}_{2}^{-}\end{array}$ & $\begin{array}{l}10000 \\
\mathrm{~cd} \mathrm{~m}^{-2}\end{array}$ & $\begin{array}{l}\text { Max } \\
\text { (Average) }\end{array}$ & $\begin{array}{l}1000 \\
\mathrm{~cd} \\
\mathrm{~m}^{-2}\end{array}$ & $\begin{array}{l}5000 \\
\mathrm{~cd} \\
\mathrm{~m}^{-2}\end{array}$ & $\begin{array}{l}10000 \\
\mathrm{~cd} \mathrm{~m}^{-2}\end{array}$ \\
\hline $\begin{array}{l}\text { A } \\
\text { (2PXZBN) }\end{array}$ & $\begin{array}{l}108.8 \\
(107.1 \pm 1.0)\end{array}$ & 75.1 & 40.9 & $\begin{array}{l}123.4 \\
(120.3 \pm 1.3)\end{array}$ & 55.8 & 20.7 & $\begin{array}{l}30.7 \\
(29.95 \pm 0.30)\end{array}$ & 24.0 & 15.8 & 11.6 \\
\hline $\begin{array}{l}\text { B } \\
(2 \mathrm{PTZBN})\end{array}$ & $\begin{array}{l}124.9 \\
(121.6 \pm 1.7)\end{array}$ & 94.4 & 55.1 & $\begin{array}{l}157.7 \\
(154.8 \pm 4.1)\end{array}$ & 79.1 & 31.9 & $\begin{array}{l}34.6 \\
(33.83 \pm 0.83)\end{array}$ & 29.5 & 20.1 & 15.4 \\
\hline $\begin{array}{l}\text { C } \\
\text { (BNSSe) }\end{array}$ & $\begin{array}{l}124.2 \\
(121.2 \pm 1.8)\end{array}$ & 110.8 & 64.5 & $\begin{array}{l}156.2 \\
(152.0 \pm 3.1)\end{array}$ & 90.2 & 40.4 & $\begin{array}{l}35.7 \\
(35.02 \pm 0.44)\end{array}$ & 32.0 & 24.0 & 18.9 \\
\hline $\begin{array}{l}\text { D } \\
\text { (BNSeSe) }\end{array}$ & $\begin{array}{l}121.0 \\
(119.7 \pm 0.8)\end{array}$ & 111.0 & 70.8 & $\begin{array}{l}146.3 \\
(147.6 \pm 2.5)\end{array}$ & 90.1 & 45.4 & $\begin{array}{l}36.8 \\
(36.40 \pm 0.22)\end{array}$ & 34.0 & 26.9 & 21.9 \\
\hline $\begin{array}{l}\mathrm{E}(\mathrm{HF} \\
\mathrm{BN3})\end{array}$ & $\begin{array}{l}164.5 \\
(162.4 \pm 2.0)\end{array}$ & 131.3 & 94.7 & $\begin{array}{l}205.8 \\
(197.7 \pm 8.7)\end{array}$ & 112.2 & 56.9 & $\begin{array}{l}40.5 \\
(40.03 \pm 0.40)\end{array}$ & 32.4 & 26.1 & 23.3 \\
\hline F (BN3) & $\begin{array}{l}152.2 \\
(147.6 \pm 3.5)\end{array}$ & 82.2 & 38.1 & $\begin{array}{l}170.7 \\
(165.6 \pm 3.9)\end{array}$ & 63.5 & 18.2 & $\begin{array}{l}38.7 \\
(36.94 \pm 1.03)\end{array}$ & 20.4 & 11.9 & 9.4 \\
\hline
\end{tabular}

${ }^{a}$ Maximum CE, and value at 1000 and $10000 \mathrm{~cd} \mathrm{~m}^{-2}$, respectively. ${ }^{\mathrm{b}}$ Maximum PE, and value at 1000 and $10000 \mathrm{~cd} \mathrm{~m}^{-2}$, respectively. ${ }^{c}$ Maximum EQE, and value at 1000, 5000 and $10000 \mathrm{~cd} \mathrm{~m}^{-2}$, respectively. The average device parameters in parentheses are based on the measurement of over fifteen independent devices.

Due to the short delayed fluorescence lifetime, high PLQY and outstanding EL performance, we anticipate BNSeSe should be a perfect candidate of TADF sensitizer for low-energy emitter. To prove it, one yellow emission MR-TADF emitter BN3 ${ }^{43}$ was chosen as the terminal emitter and the device (E) was fabricated with the following device configuration: ITO/TAPC (30 nm)/TCTA (15 $\mathrm{nm}) / \mathrm{mCBP}(10 \mathrm{~nm}) / \mathrm{EML}$ (1wt\% BN3 and 25wt\% BNSeSe in DMIC-TRZ, $50 \mathrm{~nm}) /$ POT2T $(20 \mathrm{~nm}) /$ ANT-BIZ (30 nm)/Liq (2 nm)/Al (100 $\mathrm{nm}$ ). For comparison, sensitizer-free device $\mathrm{F}$ was also fabricated with $1 \mathrm{wt} \%$ BN3 doped in DMIC-TRZ as EML. Compared to device $\mathrm{F}$, the BNSeSe sensitized device E depict lower turn-on voltage below $2.4 \mathrm{~V}$, this should be attributed to the better energy alignment between BNSeSe and DMIC-TRZ. As collected in Table 2, excellent EL performance of device F was observed with $\mathrm{EQE}_{\max }$ of $38.7 \%$, $\mathrm{CE}_{\max }$ of $152.2 \mathrm{~cd} \mathrm{~A}^{-1}, \mathrm{PE}_{\max }$ of $170.7 \mathrm{Im} \mathrm{W}^{-1}$ and maximum luminance $\left(\mathrm{L}_{\max }\right)$ of $154424 \mathrm{~cd} \mathrm{~m}^{-2}$. However, device $\mathrm{F}$ shows obvious efficiency roll-off at high luminance, EQE sudden drops to $20.4 \%$ at $1000 \mathrm{~cd} \mathrm{~m}^{-2}$ and only 11.9 at $5000 \mathrm{~cd} \mathrm{~m}^{-2}$. In sharp contrast, after introducing BNSeSe as TADF sensitizer in the EML, device E exhibits higher EQEs as well as much smaller efficiency roll off than device F. Enhanced $C E_{\max }$ of $164.5 \mathrm{~cd} \mathrm{~A}^{-1}, P E_{\max }$ of $205.8 \mathrm{Im} \mathrm{W}^{-1}$ and $L_{\max }$ of $191023 \mathrm{~cd} \mathrm{~m}^{-2}$ were observed for device E. Such high values are never reported in TADF OLEDs. More exhilaratingly, the $E_{\mathrm{m}} \mathrm{E}_{\max }$ of device $\mathrm{E}$ is boosted to $40.5 \%$ and the EQEs remain $32.4 \%$ at $1000 \mathrm{~cd} \mathrm{~m}^{-2}$ and $26.1 \%$ at $5000 \mathrm{~cd} \mathrm{~m}^{-2}$. Even at the very high brightness of $10000 \mathrm{~cd} \mathrm{~m}^{-2}$, device $E$ remains a high EQE of 23.3\%. The emission luminescence decreased from $1000 \mathrm{~cd} \mathrm{~m}^{-2}$ to $500 \mathrm{~cd} \mathrm{~m}^{-2}$ for $51 \mathrm{~h}$ without emission color change. Besides, the FWHM of device $E$ remains $40 \mathrm{~nm}$ at a brightness of $1000 \mathrm{~cd} \mathrm{~m}^{-2}$ with marginal enlargement compared to device $F$. The state-of-theart performance of BNSeSe sensitized device suggests our selenium-incorporated MR-TADF material can be ideal sensitizer for other MR-TADF emitters with long delayed lifetime. Notably, this is the first example to employ MR-TADF sensitizer for OLED, which will contribute significantly to practical applications.

\section{Conclusion}

In conclusion, we have synthesized two novel MR-TADF emitters of BNSSe and BNSeSe containing heavy-atom Se. Due to ultrastrong SOC caused by Se, RISC for BNSeSe was promoted to reach a record level (with $k_{\text {RISC }}$ valued at $2.0 \times 10^{6} \mathrm{~s}^{-1}$ ) among all 
reported MR-TADF materials. Device based on BNSeSe display excellent EL performances including high EQE $E_{\max } / \mathrm{CE}_{\max } / \mathrm{PE}_{\max }$ of $36.8 \% / 121.0 \mathrm{~cd} \mathrm{~A}^{-1} / 146.3 \mathrm{Im} \mathrm{W}^{-1}$ and ultra-low efficiency roll-off with an EQE of 21.9\% even at a high brightness of $10000 \mathrm{~cd} \mathrm{~m}^{-2}$. Furthermore, MR-TADF material (BNSeSe) was firstly used as assist dopant to sensitize the low-energy MR-TADF emitter (BN3), the hyperfluorescence OLED exhibit striking $\mathrm{EQE}_{\max }$ beyond $40 \%$ and ultra-low efficiency roll-off. Our results clearly suggest the strong heavy-atom effect could effectively promote the RISC process in MR-TADF compounds while maintaining high $\Phi_{\mathrm{PL}} \mathrm{S}$ and narrowband emission. This work sheds new light on MR-TADF emitters or sensitizers towards highly efficient OLEDs.

\section{References}

1. Hatakeyama, T. et al. Ultrapure blue thermally activated delayed fluorescence molecules: efficient HOMO-LUMO separation by the multiple resonance effect. Adv. Mater. 28, 2777-2781 (2016).

2. Suresh, S. M., Hall, D., Beljonne, D., Olivier, Y. \& Zysman-Colman, E. Multiresonant thermally activated delayed fluorescence emitters based on heteroatom-doped nanographenes: recent advances and prospects for organic light-emitting diodes. Adv. Funct. Mater. 30, 1908677-1908701 (2020).

3. Kondo, Y. et al. Narrowband deep-blue organic light-emitting diode featuring an organoboron-based emitter. Nat. Photon. 13, 678$682(2019)$.

4. Liang, X. et al. Peripheral amplification of multi-resonance induced thermally activated delayed fluorescence for highly efficient OLEDs. Angew. Chem. Int. Ed. 57, 11316-11320 (2018).

5. Zhang, Y. et al. Multi-resonance induced thermally activated delayed fluorophores for narrowband green OLEDs. Angew. Chem. Int. Ed. 58, 16912-16917 (2019).

6. Yang, M., Park, I. S. \& Yasuda, T. Full-color, narrowband, and high-efficiency electroluminescence from boron and carbazole embedded polycyclic heteroaromatics. J. Am. Chem. Soc. 142, 19468-19472 (2020).

7. Xu, Y. et al. Highly efficient electroluminescent materials with high color purity based on strong acceptor attachment onto B-Ncontaining multiple resonance frameworks. CCS Chem. 3, 2077-2091 (2021).

8. Liu, Y., Xiao, X., Ran, Y., Bin, Z. \& You, J. Molecular design of thermally activated delayed fluorescent emitters for narrowband orange-red OLEDs boosted by a cyano-functionaliztion strategy. Chem. Sci. 12, 9408-9412 (2021).

9. Zhang, Y. et al. Multi-resonance deep-red emitters with shallow potential-energy surfaces to surpass energy-gap law**. Angew. Chem. Int. Ed. https://doi.org/10.1002/anie.202107848 (2021).

10. Qi, Y. et al. Perpheral decoration of multi-resonance molecules as a versatile approach for simultaneous long-wavelength and narrowband emission. Adv. Funct. Mater. 31, 2102017-2102023 (2021).

11. Uoyama, H., Goushi, K., Shizu, K., Nomura, H. \& Adachi, C. Highly efficient organic light-emitting diodes from delayed fluorescence. Nature 492, 234-238 (2012).

12. Hirata, S. et al. Highly efcient blue electroluminescence based on thermally activated delayed fluorescence. Nat. Mater. 14, 330336 (2015).

13. Liu, Y., Li, C., Ren, Z. et al. All-organic thermally activated delayed fluorescence materials for organic light-emitting diodes. Nat. Rev. Mater. 3, 18020 (2018).

14. Wong, M. Y. \& Zysman-colman, E. Purely organic thermally activated delayed fluorescence materials for organic light-emitting diodes. Adv. Mater. 29, 1605444-1605497 (2017).

15. Brédas, J.-L., Beljonne, D., Coropceanu, V. \& Cornil, J. Charge-transfer and energy-transfer processes in $\pi$-conjugated oligomers and polymers: a molecular picture. Chem. Rev. 104, 4971-5004 (2004).

16. K. Schmidt, S. Brovelli, V. Coropceanu, D. Beljonne, J. Cornil, C. Bazzini, T. Caronna, R. Tubino, F. Meinardi, Z. Shuai, J.-L. Brédas, J. Phys. Chem. A 2007, 111, 10490.

17. Samanta, P. K., Kim, D., Coropceanu, V. \& Brédas, J.-L. Up-conversion intersystem crossing rates in organic emitters for thermally activated delayed fluorescence: impact of the nature of singlet vs triplet excited states. J. Am. Chem. Soc. 139, 4042-4051 (2017).

18. Etherington, M. K., Gibson, J., Higginbotham, H. F., Penfold, T. J. \& Monkman, A. P. Revealing the spin-vibronic coupling mechanism of thermally activated delayed fluorescence. Nat. Commun. 7, 13680 (2016). 
19. Kim, G. H. et al. Controlling the exciton lifetime of blue thermally activated delayed fluorescence emitters using a heteroatomcontaining pyridoindole donor moiety. Mater. Horiz. 4, 619-624 (2017).

20. Brédas, J.-L., Beljonne, D., Coropceanu, V. \& Cornil, J. Charge-transfer and energy-transfer processes in $\pi$-conjugated oligomers and polymers: a molecular picture. Chem. Rev. 104, 4971-5004 (2004).

21. Turro, N. J., Kavarnos, G. J., Cole, T., Scribe, P. \& Dalton, J. C., Molecular photochemistry. XXXIX. External heavy-atom-induced spin-obital coupling. Spectroscopic study of naphthonorbornanes. J. Am. Chem. Soc. 93, 1032-1034 (1971).

22. Zhao, W., He, Z. \& Tang, B.Z. Room-temperature phosphorescence from organic aggregates. Nat. Rev. Mater. 5, $869-885$ (2020).

23. Yang, Z. et al. Intermolecular electronic coupling of organic units for efficient persistent room-temperature phosphorescence. Angew. Chem. Int. Ed. 55, 2181-2185 (2016).

24. Yan, Z.-A., Lin, X., Sun, S., Ma, X. \& Tian, H. Activating room-temperature phosphorescence of organic luminophores via external heavy-atom effect and rigidity of ionic polymer matrix. Angew. Chem. Int. Ed. 60, 19735-19739 (2021)

25. Hua, T. et al. Heavy-atom effect promotes multi-resonance thermally activated delayed fluorescence. Chem. Eng. J. 426, 131169131177 (2021).

26. Zhang, D. et al. High-effciency fluorescent organic light-emitting devices using sensitizing hosts with a small singlet-triplet exchange energy. Adv. Mater. 26, 5050-5055 (2014).

27. Jeon, S.O., Lee, K.H., Kim, J.S. et al. High-efficiency, long-lifetime deep-blue organic light-emitting diodes. Nat. Photon. 15, 208215 (2021).

28. Chan, CY., Tanaka, M., Lee, YT. et al. Stable pure-blue hyperfluorescence organic light-emitting diodes with high-efficiency and narrow emission. Nat. Photon. 15, 203-207 (2021).

29. Baldo, M. A., Adachi, C. \& Forrest, S. R. Transient analysis of organic electrophosphorescence. . Transient analysis of triplettriplet annihilation. Phys. Rev. B 62, 10967-10977 (2000).

30. Hertel, D. \& Meerholz, K. Triplet-polaron quenching in conjugated polymers. J. Phys. Chem. B 111, 12075-12080 (2007).

31. Giebink, N. C. \& Forrest, S. R. Temporal response of optically pumped organic semiconductor lasers and its implication for reaching threshold under electrical excitation. Phys. Rev. B 79, 073302-073305 (2009).

32. Murawski, C., Leo, K. \& Gather, M. C. Efficiency roll-off in organic light-emitting diodes. Adv. Mater. 25, 6801-6827 (2013).

33. Einzinger, M. et al. Shorter exciton lifetimes via an external heavy-atom effect: alleviating the effects of bimolecular processes in organic light-emitting diodes. Adv. Mater. 29, 1701987-1701996 (2017).

34. Wang, D., Cheng, C., Tsuboi, T. \& Zhang, Q. Degradation mechanisms in blue organic light-emitting diodes. CCS Chem. 2, 12781296 (2020).

35. Zhang, D. et al. Efficient and stable deep-blue fluorescent organic light-emitting diodes employing a sensitizer with fast triplet upconversion. Adv. Mater. 32, 1908355-1908343 (2020).

36. Zhan, L. et al. Copper(I) complex as sensitizer enables high-performance organic light-emitting diodes with very low efficience roll-off. Adv. Funct. Mater. 2106345 (2021).

37. Kleinschmidt, M., Wüllen, C. van \& Marian, C. M. Intersystem-crossing and phosphorescence rates in fac-IrlI(ppy) 3 : a theoretical study involving multi-reference configuration interaction wavefunctions. J. Chem. Phys. 142, $094301-094316$ (2015).

38. Li, E. Y.-T., Jiang, T.-Y., Chi, Y. \& Chou, P.-T. Semi-quantitative assessment of the intersystem crossing rate: an extension of the ElSayed rule to the emissive transition metal complexes. Phys. Chem. Chem. Phys. 16, 26184-26192 (2014).

39. Zhang, Y. et al. Achieving pure green electroluminescence with CIEy of 0.69 and EQE of $28.2 \%$ from an aza-fused multiresonance emitter. Angew. Chem. Int. Ed. 59, 17499-17503 (2020).

40. Xu, Y. et al. Constructing charge-transfer excited states based on frontier molecular orbital engineering: narrowband green electroluminescence with high color purity and efficiency. Angew. Chem. Int. Ed. 59, 17442-17446 (2020).

41. Braveenth, R. et al. Achieving narrow FWHM and high EQE over 38\% in blue OLEDs using rigid heteroatom-based deep blue TADF sensitized host. Adv. Funct. Mater. 2105805 (2021).

42. Jiang, P. et al. Quenching-resistant multi-resonance TADF emitter realizes $40 \%$ extaernal quantum efficiency in narrowband electroluminescence at high doping level. Adv. Mater. https://doi.org/10.1002/adma.202106954 (2021). 
43. Qi, Y., Ning, W., Zou, Y., Cao, X., Gong, S. \& Yang, C. Peripheral decoration of multi-resonance molecules as a versatile approach for simultaneous long-wavelength and narrowband emission. Adv. Funct. Mater. 31, 2102017-2102023 (2021).

\section{Declarations}

\section{Acknowledgements}

This work was funded by the National Natural Science Foundation of China (52130308), the Shenzhen Science and Technology Program (KQTD20170330110107046) and the China Postdoctoral Science Foundation (2021 M692183). The authors also thank the Instrumental Analysis Center of Shenzhen University for analytical support.

\section{Author contributions}

C.Y. supervised the projects. C.Y., Y.X.H., Z.H. and Y.Z. designed the TADF emitters. Y.X.H., T.H. and Y.Q. synthesized emitters. Y.X.H. characterized the emitters and measured the photophysical and electrochemical properties. J.M. and H.X. fabricated the OLED devices, measured the electroluminescence and prepared thin films. Y.X.H. and H.L. performed theoretical calculations. Y.Q. conducted the transient PL measurements. Y.X.H., X.C. and C.Y. contributed to the manuscript writing. Y.X.H., H.L., J.M. and C.Y. contributed to discussions. All authors discussed the progress of the research and reviewed the manuscript.

\section{Competing interests}

SZU has field patent applications on materials and devices.

\section{Methods}

Theoretical calculations. Quantum chemical calculations were performed by the Gaussian 09 program package, density functional theory (DFT) using the B3LYP/6-31G(d,p) was performed for achieved optimized molecular geometries. Based on the optimized geometric configurations, the highest occupied molecular orbital (HOMO) and the lowest unoccupied molecular orbital (LUMO) were obtained logically. Time-dependent DFT (TD-DFT) calculations were performed at the B3LYP/6-31G(d,p) level to obtain the vertical transitions and NTOs natural transition orbitals of the singlet and triplet states based on the corresponding $\mathrm{S}_{0}$ geometries. The SOC (spin-orbit coupling) calculations were further evaluated under PySOC method.

Photophysical measurements. UV-vis absorption spectra and photoluminescence (PL) spectra were measured by a Shimadzu UV2700 spectrophotometer (Shimadzu, Japan) and a Hitachi F-7100 fluorescence spectrophotometer (Hitachi, Japan), respectively. Phosphorescence spectra (Phos) were recorded on the Hitachi F-7100 fluorescence spectrophotometer at $77 \mathrm{~K}$. The transient PL decay curves were obtained by FluoTime 300 (PicoQuant $\mathrm{GmbH}$ ) with a Picosecond Pulsed UV-LASTER (LASTER375) as the excitation source. The photoluminescence quantum yields ( $\Phi_{\mathrm{PL}} \mathrm{S}$ or PLQYs) were measured with a Hamamatsu UV-NIR absolute PL quantum yield spectrometer (C13534, Hamamatsu Photonics) equipped with a calibrated integrating sphere, the integrating sphere was purged with dry argon to maintain an inert atmosphere.

Device fabrication and performance measurements. The ITO coated glass substrates with a sheet resistance of $15 \Omega$ square ${ }^{-1}$ were consecutively ultrasonicated with acetone/ethanol and dried with nitrogen gas flow, follwed by 20 min ultraviolet light-ozone (UVZ) treatment in a UV-ozone surface processor (PL16 series, Sen Lights Corporation). Then the sample was transferred to the deposition system. Both 8-hydroxyquinolinolato-lithium (Liq) as electron injection layer and aluminum (al) as cathode layer were deposited by thermal evaporation at $5 \times 10^{-5} \mathrm{~Pa}$. Additionally, the organic layers were deposited at the rates of $0.2-3 \AA / \mathrm{s}$. After the organic film deposition, Liq and Al layer were deposited with rates of 0.1 and $3 \AA / s$, respectively. The emitting area of the device is about 0.09

$\mathrm{cm}^{2}$. The current density-voltage-luminance $(J-V-L), L-E Q E$ curves and electroluminescence spectra were measured using a Keithley 2400 source meter and an absolute EQE measurement system (C9920-12, Hamamatsu Photonics, Japan).

\section{Figures}


a

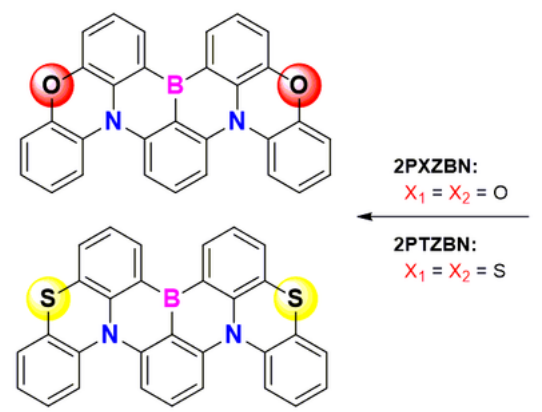

Limited SOC

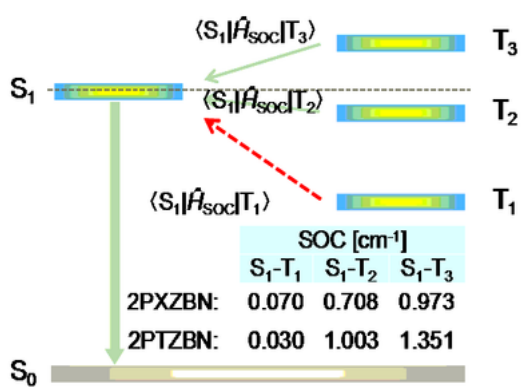

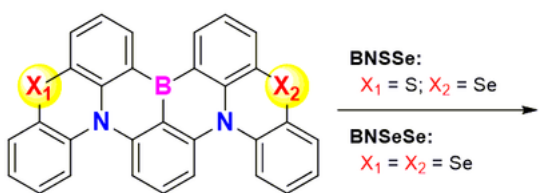
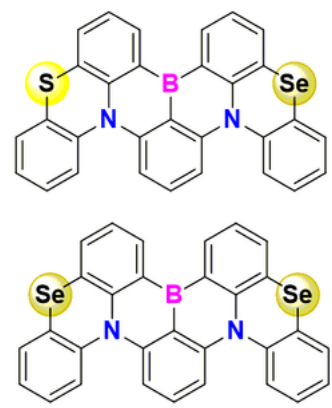

Ultra-strong SOC

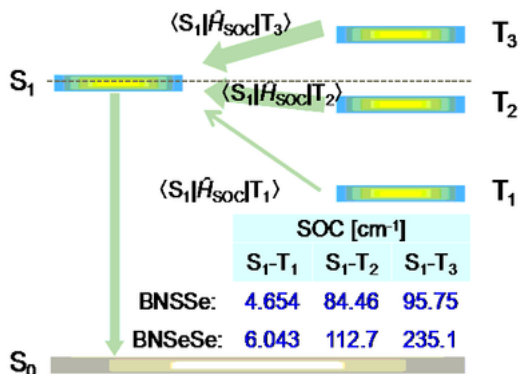

b
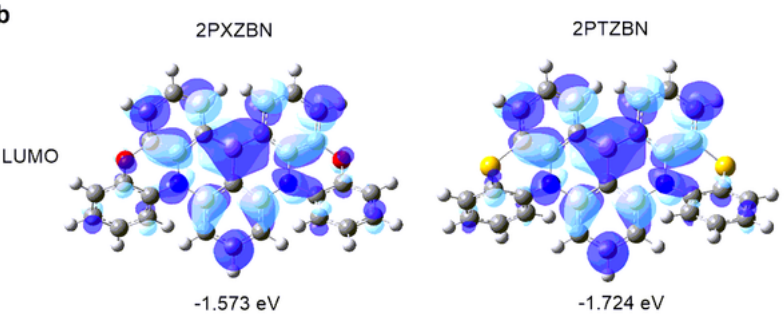

$$
\left\{\begin{array}{c}
E_{g}=3.280 \mathrm{eV} \\
f=0.2215 \\
\Delta E_{\mathrm{ST}}=0.4462 \mathrm{eV}
\end{array}\right.
$$
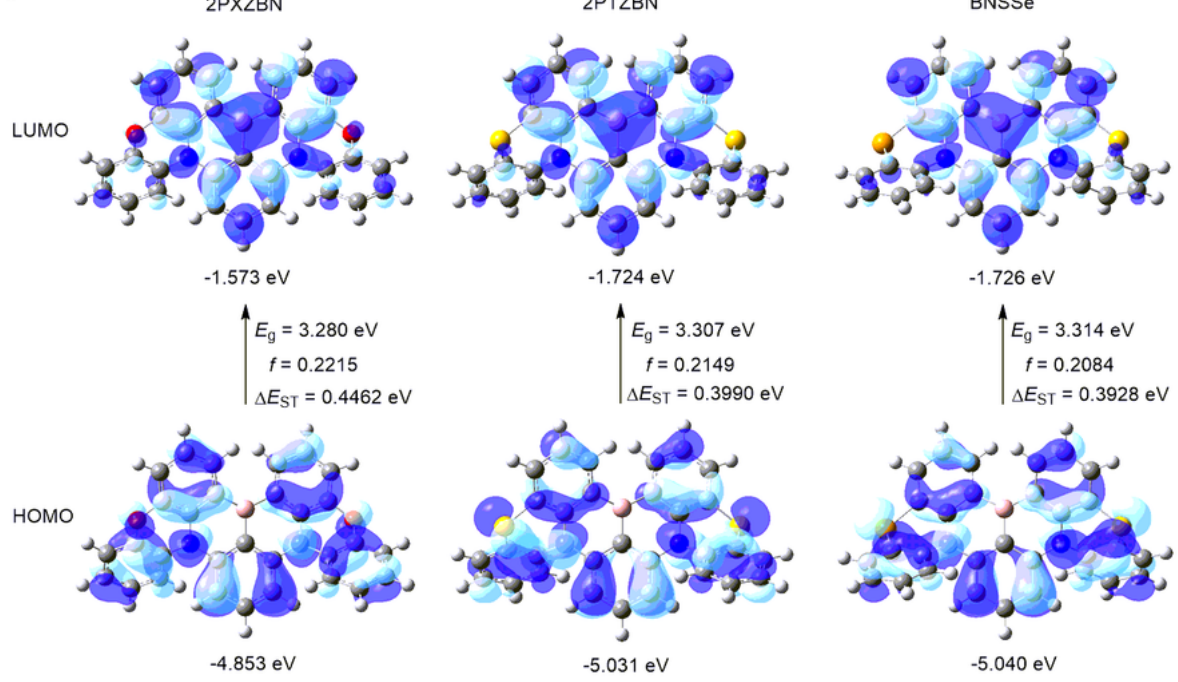

$-1.726 \mathrm{eV}$

$\left\{\begin{array}{c}E_{g}=3.314 \mathrm{eV} \\ f=0.2084 \\ \Delta E_{S T}=0.3928 \mathrm{eV}\end{array}\right.$
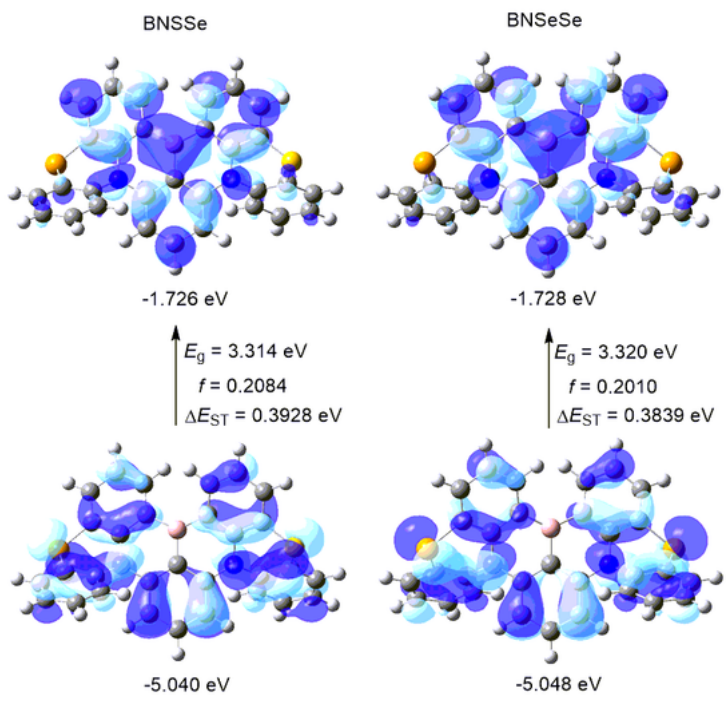

Figure 1

Molecular design. a, Molecular structures and the SOC constants of 2PXZBN, 2PTZBN, BNSSe and BNSeSe, respectively. b, DFTcalculated HOMO and LUMO distributions, energy levels, energy band gaps and oscillator strengths of 2PXZBN, 2PTZBN, BNSSe and BNSeSe, respectively. 

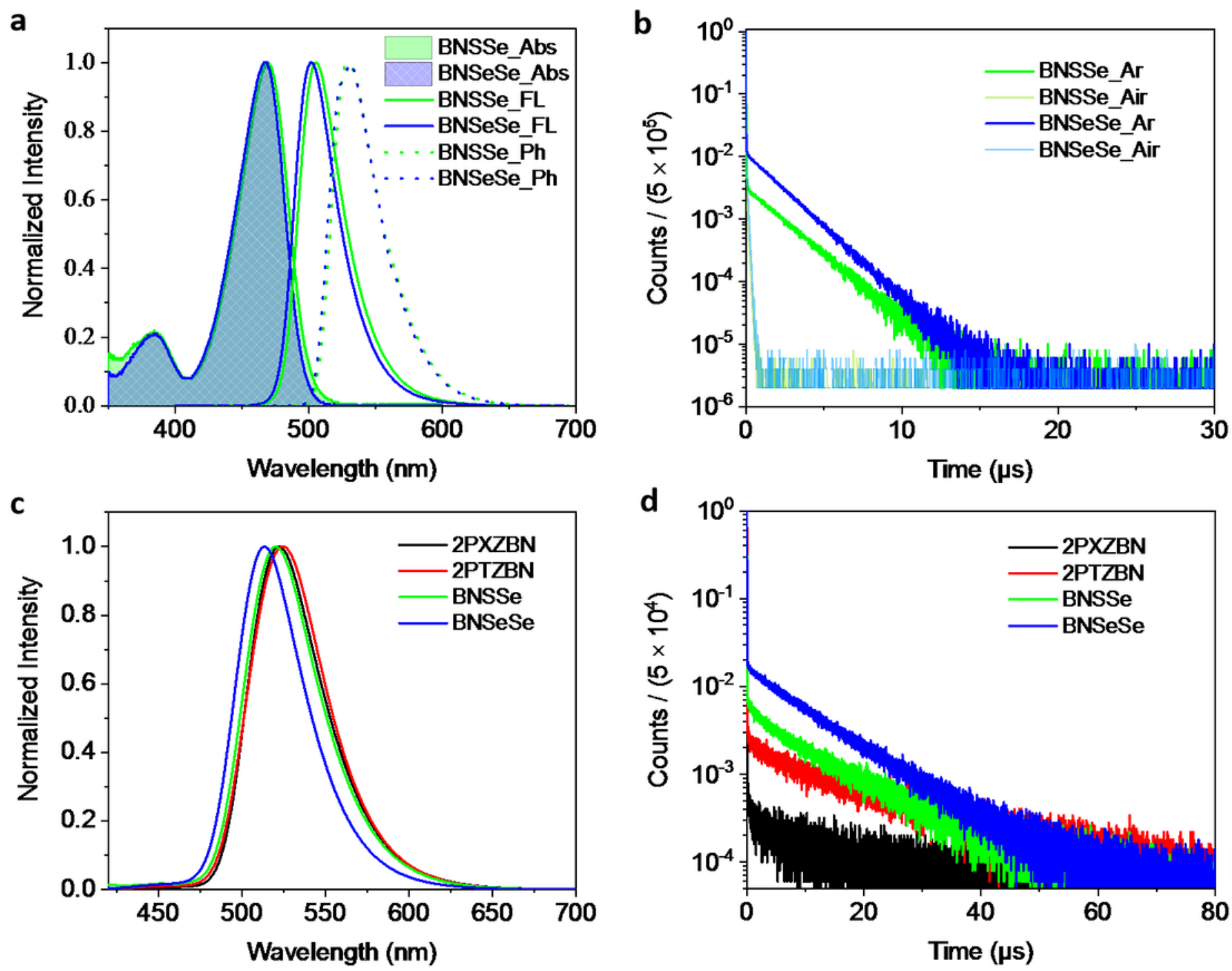

Figure 2

Molecular design. a, UV-vis absorption, fluorescence spectra (298 K) and phosphorescence spectra (77 K) of BNSSe and BNSeSe in toluene solution. b, Transient PL decay curves of BNSSe and BNSeSe in toluene solution before and after Ar gas bubbling for 30 min. c, PL spectra of 2PXZBN, 2PTZBN, BNSSe and BNSeSe in 1wt\% DMIC-TRZ doped film. d, Transient PL decay curves of 2PXZBN, 2PTZBN, BNSSe and BNSeSe in 1wt\% DMIC-TRZ doped film. 
a

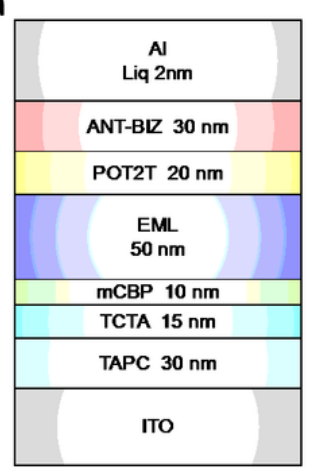

C

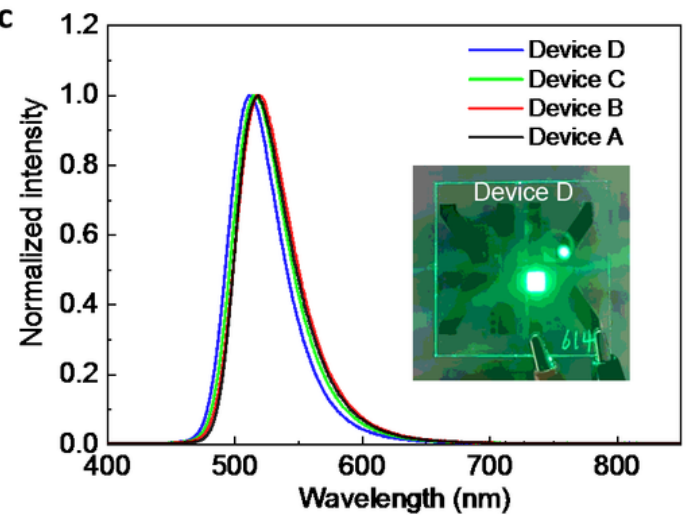

e

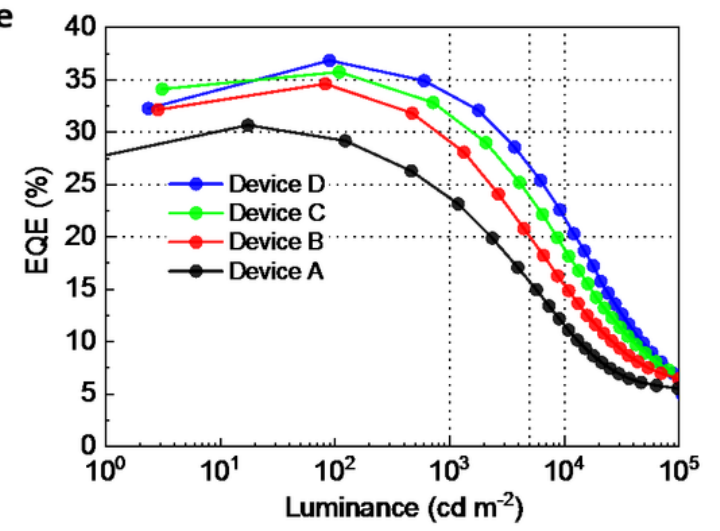

b

Device A: 1wt\% 2PXZBN : DMIC-TRZ

Device B: 1wt\% 2PTZBN : DMIC-TRZ

Device C: 1wt\% BNSSe : DMIC-TRZ

Device D: 1wt\% BNSeSe : DMIC-TRZ

Device F: 1wt\% BN3 : DMIC-TRZ

Device E (hyperfluorescence OLED):

1wt\% BN3 : 25wt\% BNSeSe : DMIC-TRZ
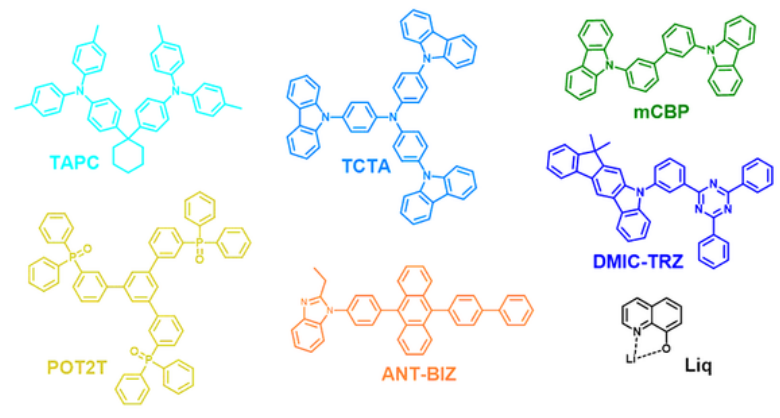

Liq

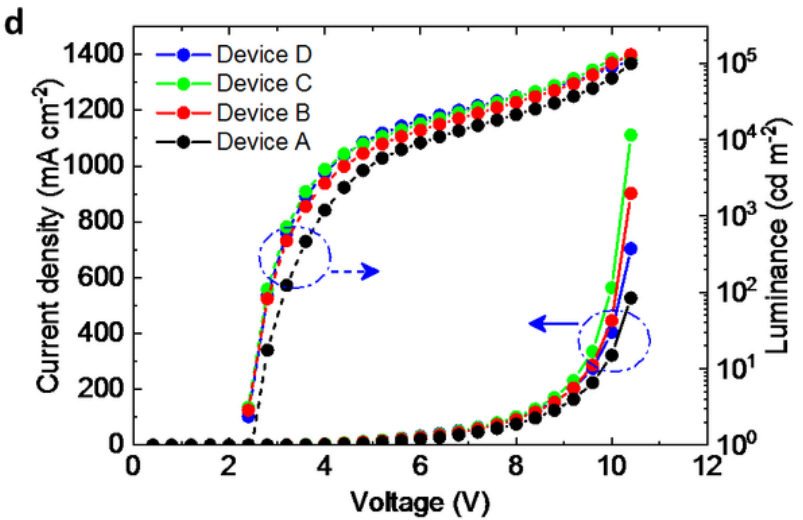

f

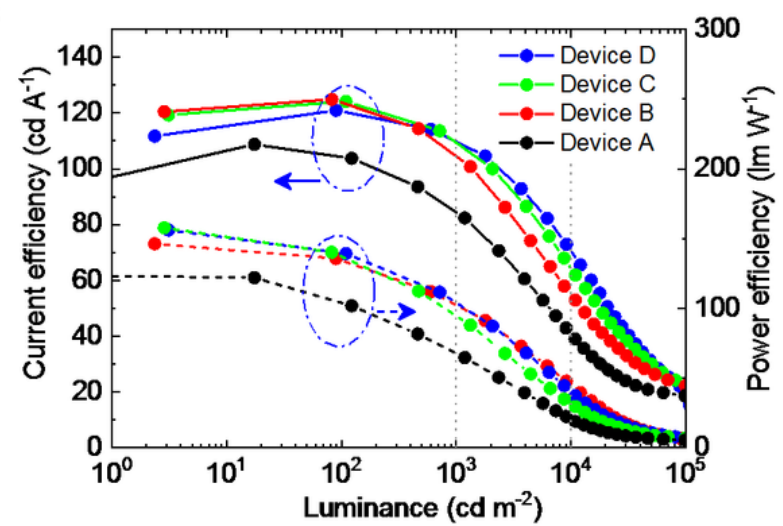

Figure 3

OLED A-D. a, Device structure. b, the chemical structures used for the respective layers. c, Normalized EL spectra of 2PXZBN, 2PTZBN, BNSSe and BNSeSe, respectively. Inset: the photograph of device D. d, Current density and luminance versus driving voltage characteristics. e, EQE versus luminance characteristics. f, Current and power efficiency versus luminance characteristics. 
a

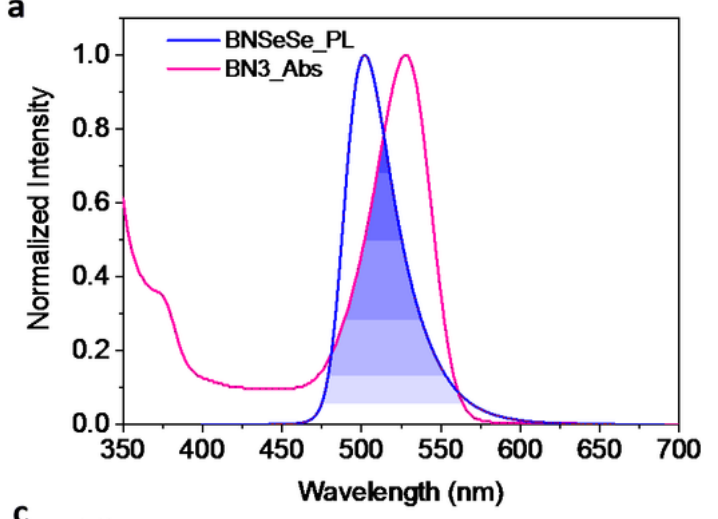

C

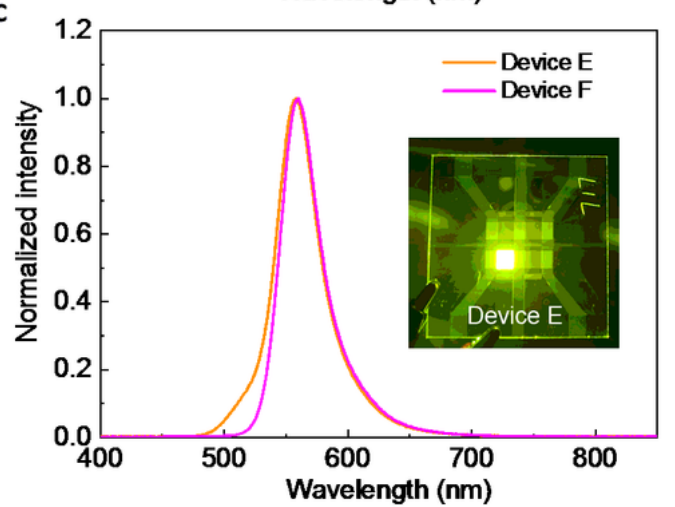

e

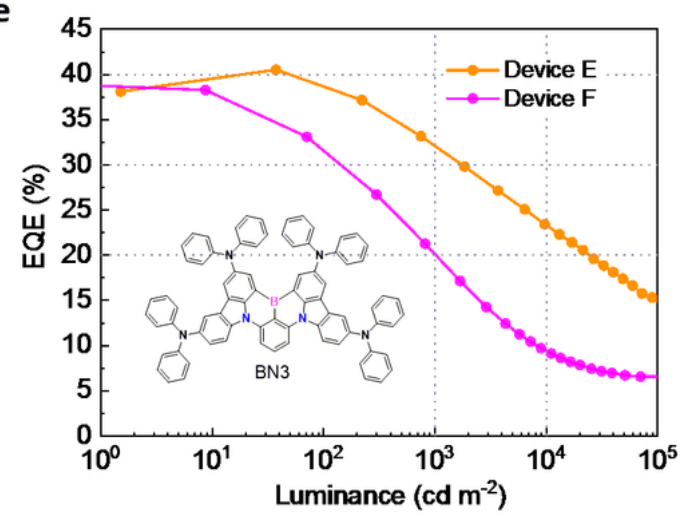

b

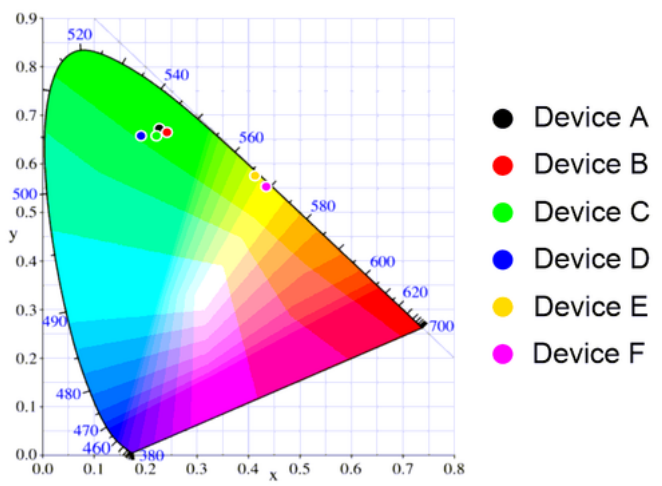

d

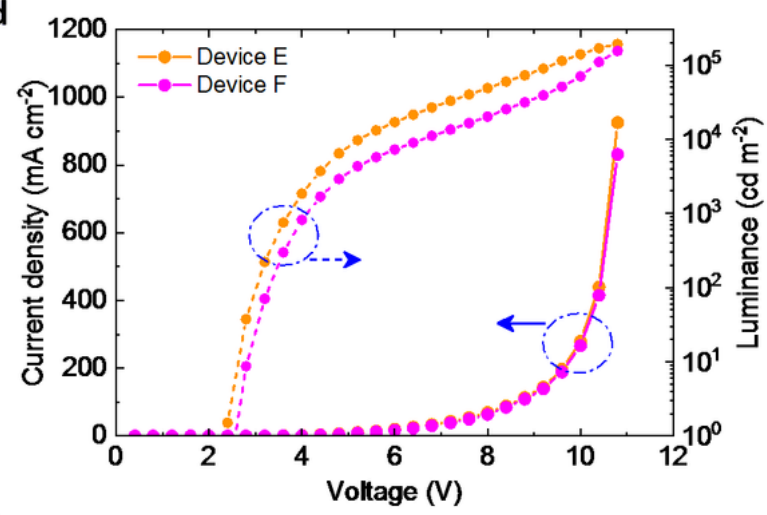

f

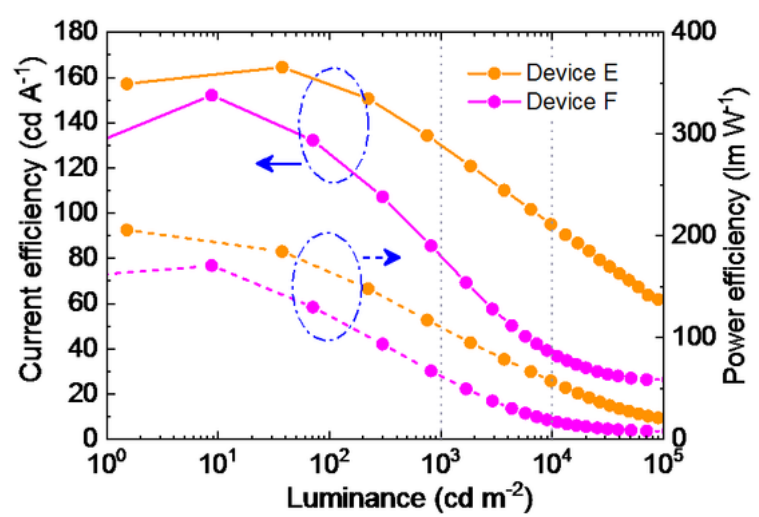

Figure 4

HF OLED . a, Emission spectrum of BNSeSe and UV-vis absorption of BN3 in solution. Filled area reveals the overlap of them. $\mathbf{b}$, EL color coordinates of Device A-F in the CIE 1931 chromaticity diagram. c, Normalized EL spectra of BN3 in Device E and F, respectively. Inset: photograph of device E. d, Current density and luminance versus driving voltage characteristics. e, EQE versus luminance characteristics. f, Current and power efficiency versus luminance characteristics.

\section{Supplementary Files}

This is a list of supplementary files associated with this preprint. Click to download.

- Supportinginformation.docx 